\section{Pneumologie}

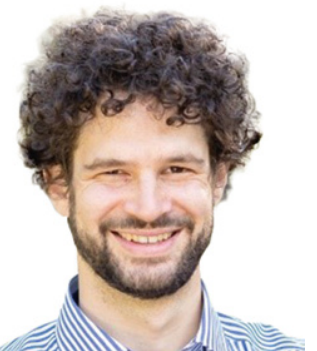

Stephan Pramsohler

Hermann Buhl Trainings- und Forschungszentrum, Bad Aibling, Deutschland

\title{
Stephan Pramsohler
}

\section{Träger des «Doktorandenpreises Pneumologie der Deutschen Lungenstiftung» 2020}

\section{Forschungsschwerpunkte}

- Hypoxie und Trainingsforschung

\section{Akademischer Werdegang}

- seit 3/2010: Klettern mit Patienten mit multipler Sklerose am Kuratorium für Prävention und Rehabilitation der TU München

• 10/2006-09/2011: Sportstudium an der technischen Universität München mit der Fachrichtung Prävention und Rehabilitation

- 03/2013-06/2018: Doktorand und wissenschaftlicher Mitarbeiter am Hermann Buhl Institut für Hypoxie und Schlafmedizinforschung in Bad Aibling

\section{Lehre}

- seit 07/2012: Hypoxie- und Höhenmedizin, Universität Ulm, Abteilung Medizin

- seit 03/2017: Neue Methoden in der Trainingstherapie, Universität Innsbruck, Institut für Sportwissenschaft

\section{Homepage \\ -www.hypoxie.com}

Welche Fragestellung lag Ihrer ausgezeichneten klinischen Arbeit «Physiological effects of training in hypoxic environments and its benefits for prevention and rehabilitation» zugrunde?

Seit Langem ist bekannt, dass Sauerstoffmangel (Hypoxie) negative Reaktionen im menschlichen Körper auslöst. Spätestens seit den olympischen Spielen 1968 in Mexico City ist allerdings auch bekannt, dass Sauerstoffmangel ebenso positive Prozesse im Körper auslösen kann. Es scheint also, wie so oft in der Medizin, die Frage der Dosis den Unterschied zwischen Gift und Medizin zu bedingen. Im Fokus meiner Arbeit stand daher die Abwägung von Risiken und die Einsetzbarkeit von Training in hypoxischen Umgebungen als Therapeutikum bei geriatrischen Patienten.

Dazu waren zunächst mehrere Schritte nötig. Zum einen musste geklärt werden, welche Risiken die Hypoxie mit sich bringt und wir mussten eine Möglichkeit zur technischen Umsetzung des Trainings mit geriatrischen Patienten finden. Normobare Hypoxie in einem Trainingsinstitut bot dafür die idealen Voraussetzungen. Daraus ergab sich aber die zusätzliche Fragestellung, welche Unterschiede zwischen normobarer Hypoxie (normaler Umge-
Dr. Stephan Pramsohler

Bummstr. 1

80804 München

s.pramsohler@gsund.bayern 
bungsdruck und reduzierter Sauerstoffgehalt) und hypobarer Hypoxie (verringerter Umgebungsdruck, gleicher Sauerstoffprozentanteil) bestehen. Dazu führten wir eine Vergleichsstudie in «echter» Höhe auf dem Mauna Kea durch und verglichen die Daten mit dem Laborversuch. Der Mouna Kea auf Hawaii eignete sich dafür besonders gut, da der Gipfel trotz einer Höhe von 4205 m noch mit dem Auto erreichbar ist und wir dadurch das Equipment leichter in diese Höhe bringen konnten.

Welche Herausforderungen stellten sich Ihnen bei der Konzeption und/oder Entwicklung Ihres Forschungsansatzes?

Die Arbeit mit geriatrischen Patienten fordert in so manch einer Situation eine andere Herangehensweise als die Arbeit mit jüngeren. Gut etablierte Messverfahren wie zum Beispiel die Spiroergometrie zeigen bei solchen Patientengruppen schneller ihre Schwächen. Dieser Umstand forderte eine genaue und vorausschauende Planung. Zusätzlich stellte uns die Planung des Feldversuches mit der Besteigung des Mouna Kea in Hawaii vor ungeahnte und auch fachfremde Problematiken. Hier musste zum Beispiel mit der Firma für das Kalibrationsgas verhandelt werden, wie eine Lieferung nach Hawaii möglich gemacht werden kann. Außerdem mussten wir das Höhenprofil der Wanderung mit Steigungswerten und Geschwindigkeiten in ein Profil für die Laufbänder umrechnen und die Umgebungsbedingungen sowie den Ernährungsstatus unserer Probanden im Labor exakt nachstellen. Die Messungen auf 4205 m über dem Meeresspiegel durchzuführen, stellte Untersucher und auch das Equipment vor große Herausforderungen.

\section{Was hat Sie an den Ergebnissen Ihrer Untersuchung am meisten überrascht?}

Insbesondere überraschte mich, wie begeisterungsfähig Patienten, die teilweise über 90 Jahre alt waren, für die Forschung waren. Dieser Umstand im Zusammenhang mit den tollen Ergebnissen haben mir gezeigt, dass Höhentraining als Therapieform tatsächlich eine Chance hat.

In welcher Weise sind Ihre Erkenntnisse für die klinische Praxis besonders relevant?

«High Intensity Training» hat in den vergangenen Jahren gezeigt, dass die positiven Effekte auf den Körper denen von moderatem
Ausdauertraining vielfach überlegen sind. Dieses Training ist aber durch die hohen Intensitäten und Kräfte, die damit auf den Körper wirken, bisher nur für vollkommen Gesunde zu empfehlen. Mit Unterstützung von Höhenluft kann diese Trainingsform nun auch in Präventions- und Rehabilitationsprogrammen für vulnerable Personengruppen Einzug finden.

Was ist aus Ihrer Erfahrung wichtig für die Schnittstelle zwischen Forschung und klinischer Medizin?

Der Weg von der Forschung in den klinischen Alltag ist oft sehr weit und langwierig. Um eine möglichst große Qualität und Sicherheit von Verfahren zu gewährleisten, befinden sich auf diesem Weg daher oft viele Meilensteine, die es zu überwinden gilt. Dieser Prozess hat Vor- und Nachteile und sollte regelmäßig überdacht, und wenn nötig auch gestrafft werden, um unnötige Hürden und Zwischenschritte abzubauen.

Womit schaffen Sie für sich selbst und für Ihr Umfeld einen Ausgleich zu Ihrem Einsatz in Klinik und Forschung?

Sport nimmt für mich hier einen sehr großen Stellenwert ein und ich befinde mich, wann immer es möglich ist, kletternd und wandernd in den Bergen.

Herr Dr. Pramsohler, wir bedanken uns ganz herzlich für das Interview!

\section{ANGABEN ZUM PREIS}

Die Deutsche Lungenstiftung verleiht jährlich im Rahmen der Jahrestagung der Deutschen Gesellschaft für Pneumologie und Beatmungsmedizin e.V. (DGP) den Dissertationspreis Pneumologie zur Förderung des wissenschaftlichen Nachwuchses. Der Preis wird von der Firma Boehringer Ingelheim Pharma GmbH \& Co. KG gestiftet und zeichnet die beste klinische und die beste experimentelle Dissertationsarbeit auf dem Gesamtgebiet der Pneumologie aus. Der Preis ist mit jeweils 3000 EUR dotiert.

www.lungenstiftung.de/aktivitaeten/dissertationspreis-pneumologie 